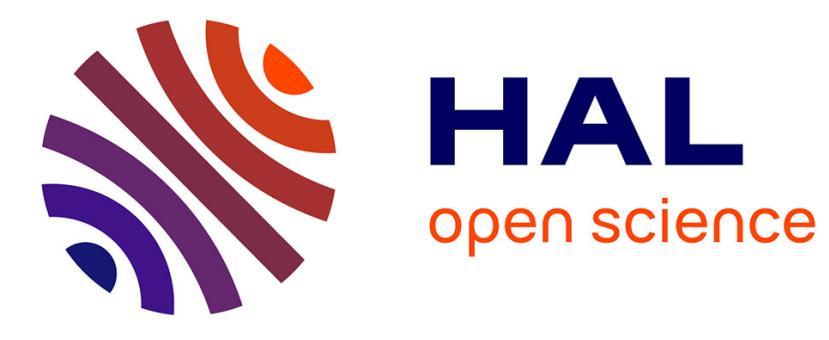

\title{
Topological properties of thinning in 2-D pseudomanifolds
}

Nicolas Passat, Michel Couprie, Loïc Mazo, Gilles Bertrand

\section{To cite this version:}

Nicolas Passat, Michel Couprie, Loïc Mazo, Gilles Bertrand. Topological properties of thinning in 2-D pseudomanifolds. Journal of Mathematical Imaging and Vision, 2010, 37 (1), pp.27-39. 10.1007/s10851-010-0190-x . hal-00622471

\section{HAL Id: hal-00622471 \\ https://hal.science/hal-00622471}

Submitted on 7 Sep 2012

HAL is a multi-disciplinary open access archive for the deposit and dissemination of scientific research documents, whether they are published or not. The documents may come from teaching and research institutions in France or abroad, or from public or private research centers.
L'archive ouverte pluridisciplinaire HAL, est destinée au dépôt et à la diffusion de documents scientifiques de niveau recherche, publiés ou non, émanant des établissements d'enseignement et de recherche français ou étrangers, des laboratoires publics ou privés. 


\title{
Topological properties of thinning in 2-D pseudomanifolds
}

\author{
Nicolas Passat • Michel Couprie - Loïc Mazo • Gilles Bertrand
}

\begin{abstract}
Preserving topological properties of objects during thinning procedures is an important issue in the field of image analysis. In the case of 2-D digital images (i.e. images defined on $\mathbb{Z}^{2}$ ) such procedures are usually based on the notion of simple point. In contrast to the situation in $\mathbb{Z}^{n}, n \geq 3$, it was proved in the 80's that the exclusive use of simple points in $\mathbb{Z}^{2}$ was indeed sufficient to develop thinning procedures providing an output that is minimal with respect to the topological characteristics of the object. Based on the recently introduced notion of minimal simple set (generalising the notion of simple point), we establish new properties related to topology-preserving thinning in 2-D spaces which extend, in particular, this classical result to cubical complexes in 2-D pseudomanifolds.
\end{abstract}

Keywords topology preservation $\cdot$ simple points $\cdot$ simple sets · cubical complexes · collapse $\cdot$ confluence . pseudomanifolds

\section{Introduction}

Topological properties are fundamental in many applications of image analysis, in particular in cases where the retrieval and/or the preservation of topology of real complex structures is required. In this context, numerous methods have been developed to process discrete 2-D and 3-D binary images, essentially to perform skeletonisation, homotopic transforms or segmentation (see e.g. [1-3]).

Nicolas Passat, Loïc Mazo

Université de Strasbourg, LSIIT, UMR CNRS 7005, France

Tel.: +33-368854496

Fax: $+33-368854455$

E-mail: passat@unistra.fr

Michel Couprie, Gilles Bertrand

Université Paris-Est, Laboratoire d'informatique Gaspard-Monge, Équipe A3SI, ESIEE Paris, France
Such methods are generally based on the notion of simple point $[4,5]$. Intuitively, a point (or pixel) of a discrete object $X$ is said to be simple if it can be removed from $X$ without altering its topology.

Let us consider an object $X$, i.e. a set of points in $\mathbb{Z}^{n}$, and a subset $Y$ of $X$ called constraint set. A very common topology-preserving thinning scheme [6] consists of repeating the following steps until stability:

- choose (according to a given priority function) a point $x$ in $X \backslash Y$ that is simple for $X$;

- remove $x$ from $X$.

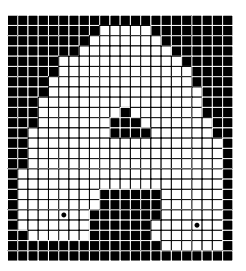

(a)

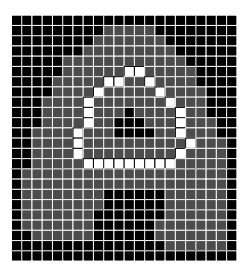

(b)

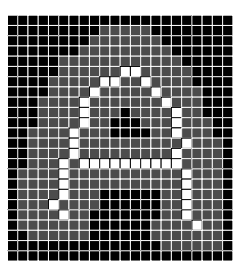

(c)
Fig. 1 (a) An object $X$ (in white) and a subset $Y$ of $X$ (two pixels marked by black dots). (b) A homotopic skeleton of $X$ (empty constraint set). (c) A homotopic skeleton of $X$ constrained by $Y$.

The result of such a procedure, called homotopic skeleton of $X$ constrained by $Y$, is a subset $Z$ of $X$, which (i) is topologically equivalent to $X$, (ii) includes $Y$ and (iii) has no simple point outside of $Y$. We show an illustration in Fig. 1, notice in particular that the constraint set is useful to preserve some geometrical characteristics of the object.

The following question is fundamental with regard to the behaviour of sequential thinning procedures:

(1) Is $Z$ always a minimal result, in the sense that it does not strictly include a subset $Z^{\prime}$ having the same properties (i), (ii) and (iii)? 
If we consider the 3-D case, the answer to this question is no. For example, if $X$ is a cuboid and $Y=\emptyset$, then, depending on the order of the point removals, the result $Z$ of the above procedure might not be composed of a single point. As pointed out recently [7], there exist various kinds of configurations in which a 3-D topology-preserving thinning algorithm can be "blocked" before reaching a minimal result.

In the discrete plane $\mathbb{Z}^{2}$, question (1) was answered positively by C. Ronse in the 80's, after a partial answer was given in the early 70's by A. Rosenfeld.

In 1970, in the same article where he introduced the notion of simple point [8], A. Rosenfeld proved that any finite subset of $\mathbb{Z}^{2}$ that is connected and has no holes, can be reduced to a single point by iterative removal of simple points, in any order. In [9], he also proved that any connected object with one hole reduces in the same way to a closed curve.

In 1986, C. Ronse introduced the notion of strong deletability in $\mathbb{Z}^{2}$ [10]. It is, to the best of our knowledge, the first attempt to explicitly ${ }^{1}$ generalise the notion of simple points to a more general notion of simple sets.

According to Def. 2.5 of [10], and skipping formal details, a subset $S$ of $X \subseteq \mathbb{Z}^{2}$ is strongly deletable from $X$ if (i) each connected component of $X$ includes exactly one connected component of $X \backslash S$, and (ii) each connected component of $\bar{X} \cup S$ includes exactly one connected component of $\bar{X}$, where $\bar{X}$ denotes the complement of $X$ in $\mathbb{Z}^{2}$.

In the same article, $C$. Ronse proposed several results related to strongly deletable sets, which can be summarised as follows (see also [12], Prop. 2.4.).

Theorem 1 (From [10], Lem. 3.1, 3.2, Prop. 3.3) Let $X \subseteq$ $\mathbb{Z}^{2}$. Let $S \subseteq X$. If $S$ is strongly deletable from $X$, then:

- there exists $x \in S$ such that $x$ is a simple point for $X$;

- for all $x \in S$ such that $x$ is a simple point for $X, S \backslash\{x\}$ is strongly deletable for $X \backslash\{x\}$.

Consequently, if $Y \subseteq X \subseteq \mathbb{Z}^{2}$ and $Y$ is topologically equivalent to $X$ (more precisely, if $X \backslash Y$ is strongly deletable from $X$ ), then $Y$ may be obtained from $X$ by iterative removal of simple points, in any arbitrary order.

To summarise, question (1) received a positive answer in $\mathbb{Z}^{2}$ and a negative one in $\mathbb{Z}^{3}$ (and also for higher dimensions). Still, there are spaces for which this question remained open until now: the case of two-dimensional structures in $n$-dimensional spaces, $n \geq 3$. Such structures are often used in practice, e.g. to represent thin objects or (parts of) boundary of objects in 3-D image analysis and in finite element modelling.

The main outcome of this article is a theorem (Th. 3) that states a property analogous to Th. 1, holding in a large

\footnotetext{
1 Note that A. Rosenfeld proved, ten years earlier [11], that the sets of points deleted by certain parallel thinning algorithms satisfy the conditions used to define strong deletability.
}

family of 2-D digital spaces, namely the pseudomanifolds (see Fig. 9 where some pseudomanifolds are depicted).

This study is developed in the framework of cubical complexes [13], in which we can retrieve and generalise the concepts of digital topology in $\mathbb{Z}^{n}$. The definition of simple sets that we use here is based on the operation of collapse, a topology-preserving transformation known in algebraic topology. This definition makes sense whatever the dimension.

The proof of Th. 3 is based on a property of collapse, that we call a confluence property (Th. 2), which is introduced and proved in this article.

Th. 3 is also closely related to the notion of minimal simple set introduced by some of the authors (see [7]), as we derive it using the following property: if $X$ is a strict subset of a pseudomanifold, then any minimal simple subset of $X$ is a simple point (Prop. 22).

Thanks to a correspondence between the notion of minimal simple set used here and the one of simple point [14], we retrieve as particular cases of Th. 3 the results of A. Rosenfeld and C. Ronse discussed before. However, the techniques of proof used in this article are essentially different from the ones used by these authors, and the generalisation of their results is not trivial.

Finally, we devote a section (Sec. 7) to a result related to parallel thinning that can be derived from Th. 3, based on the notion of critical kernel $[15,16]$.

This article is self-contained. Notice that all notions, properties and proofs presented hereafter can be easily transposed in the framework of simplicial complexes (i.e., triangulated objects).

\section{Background notions}

In this section, we provide basic definitions and properties related to the notions of cubical complexes, collapse and simple sets (the last two ones enabling to modify a complex without altering its topology), see also $[13,16,17]$.

\subsection{Cubical complexes}

If $T$ is a subset of $S$, we write $T \subseteq S$. Let $\mathbb{Z}$ be the set of integers. Let $k, \ell \in \mathbb{Z}$, we denote by $[k, \ell]$ the set $\{i \in \mathbb{Z} \mid k \leq$ $i \leq \ell\}$.

We consider the families of sets $\mathbb{F}_{0}^{1}, \mathbb{F}_{1}^{1}$, such that $\mathbb{F}_{0}^{1}=$ $\{\{a\} \mid a \in \mathbb{Z}\}$, and $\mathbb{F}_{1}^{1}=\{\{a, a+1\} \mid a \in \mathbb{Z}\}$. A subset $f$ of $\mathbb{Z}^{n}$ $(n \geq 2)$ that is the Cartesian product of $m$ elements of $\mathbb{F}_{1}^{1}$ and $n-m$ elements of $\mathbb{F}_{0}^{1}$ is called a face or an $m$-face of $\mathbb{Z}^{n}, m$ is the dimension of $f$, we write $\operatorname{dim}(f)=m$ (see Fig. 2a,b).

If $n \geq 2$, we denote by $\mathbb{F}^{n}$ the set composed of all faces of $\mathbb{Z}^{n}$.

An $m$-face of $\mathbb{Z}^{n}$ is called a point if $m=0$, a (unit) edge if $m=1$, a (unit) square if $m=2$. 
Let $f$ be a face in $\mathbb{F}^{n}$. We set $\hat{f}=\left\{g \in \mathbb{F}^{n} \mid g \subseteq f\right\}$. Any $g \in \hat{f}$ is a face of $f$ (or of $\hat{f}$ ).

If $X$ is a set of faces of $\mathbb{F}^{n}$, we write $X^{-}=\bigcup_{f \in X} \hat{f}$, and we say that $X^{-}$is the closure of $X$.

A set $X$ of faces of $\mathbb{F}^{n}$ is a cell or an $m$-cell if there exists an $m$-face $f \in X$, such that $X=\hat{f}$. The boundary of a cell $\hat{f}$ is the set $\hat{f}^{*}=\hat{f} \backslash\{f\}$ (see Fig. 2).

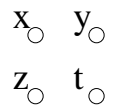

(a)

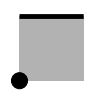

(b)

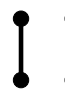

(c) (d)

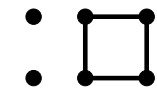

(e) (f)
Fig. 2 (a) Four points of $\mathbb{Z}^{2}: x=(0,1) ; y=(1,1) ; z=(0,0) ; t=(1,0)$. (b) A graphical representation of the set of faces $\left\{f_{0}, f_{1}, f_{2}\right\}$ in $\mathbb{F}^{2}$, where $f_{0}=\{z\}=\{0\} \times\{0\}$ (a 0 -face), $f_{1}=\{x, y\}=\{0,1\} \times\{1\}$ (a 1-face), and $f_{2}=\{x, y, z, t\}=\{0,1\} \times\{0,1\}$ (a 2-face). (c) A 1-cell $\hat{c}$. (d) A 2-cell $\hat{d}$. (e) The boundary $\hat{c}^{*}$ of $\hat{c}$. (f) The boundary $\hat{d}^{*}$ of $\hat{d}$.

A finite set $X$ of faces of $\mathbb{F}^{n}$ is a complex $\left(\right.$ in $\mathbb{F}^{n}$ ) if for any $f \in X$, we have $\hat{f} \subseteq X$.

Let $S, X$ be two sets of faces of $\mathbb{F}^{n}$. If $X$ is a complex and $X \subseteq S$, we write $X \leq S$. Furthermore, if $S$ is also a complex, then we say that $X$ is a subcomplex of $S$.

Let $X \subseteq \mathbb{F}^{n}$. A face $f \in X$ is a facet of $X$ if there is no $g \in X$ such that $f \in \hat{g}^{*}$, in other words, if $f$ is maximal for inclusion. A facet of $X$ that is an $m$-face is also called an $m$ facet of $X$. We denote by $X^{+}$the set composed of all facets of $X$ (see Fig. 3). Note that the notion of facet of a complex can intuitively be seen as the analogue of the notions of pixels and voxels in the framework of 2-D and 3-D digital topology.

If $X$ is a complex, observe that in general, $X^{+}$is not a complex, and that $\left(X^{+}\right)^{-}=X$. More generally, for any subset $Y$ of $\mathbb{F}^{n},\left(Y^{+}\right)^{-}=Y^{-}$.

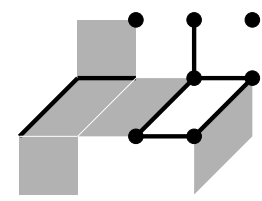

(a)

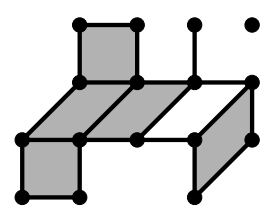

(c)

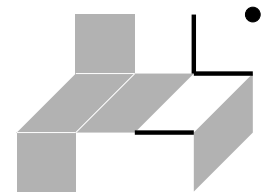

(b)

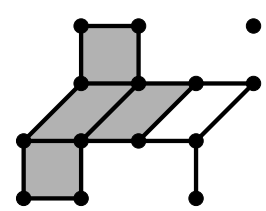

(d)
Fig. 3 (a) A set $X$ of 0-, 1- and 2-faces in $\mathbb{F}^{3}$, which is not a complex. (b) The set $X^{+}$, composed by all facets of $X$. (c) The set $X^{-}$, i.e. the closure of $X$, which is a complex. (d) A subcomplex of $X^{-}$.
Let $X \subseteq \mathbb{F}^{n}, X \neq \emptyset$. The dimension of $X$ is the number $\operatorname{dim}(X)=\max \{\operatorname{dim}(f) \mid f \in X\}$, and we set $\operatorname{dim}(\emptyset)=-1$. We say that $X$ is pure if for each $f \in X^{+}$, we have $\operatorname{dim}(f)=$ $\operatorname{dim}(X)$. Let $m$ be an integer. We say that $X$ is an $m$-complex if $X$ is a complex and $\operatorname{dim}(X)=m$. If $X$ is an $m$-complex with $m \leq 1$, then we also say that $X$ is a graph (see [18]).

Let $Y \leq X \leq \mathbb{F}^{n}$. If $Y^{+} \subseteq X^{+}$, we say that $Y$ is a principal subcomplex of $X$ and we write $Y \sqsubseteq X$ (see Fig. 4).

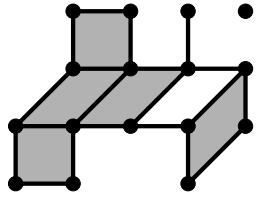

(a)

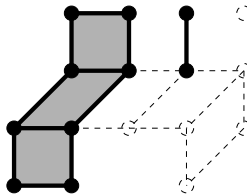

(b)

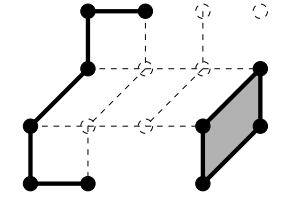

(c)
Fig. 4 (a) A complex $X$. (b) A subset $Y$ of $X$, which is a principal subcomplex of $X$ (i.e., $Y \sqsubseteq X)$. (c) A subset $Z$ of $X$, which is a subcomplex of $X$ but not a principal subcomplex of $X$.

Let $X \subseteq \mathbb{F}^{n}$. A sequence $\pi=\left\langle f_{i}\right\rangle_{i=0}^{\ell}(\ell \geq 0)$ of faces in $X$ is a path in $X$ (from $f_{0}$ to $\left.f_{\ell}\right)$ if for each $i \in[0, \ell-1]$, either $f_{i}$ is a face of $f_{i+1}$ or $f_{i+1}$ is a face of $f_{i}$; the integer $\ell$ is the length of $\pi$. The path $\pi$ is said to be closed whenever $f_{0}=f_{\ell}$, it is a trivial path whenever $\ell=0$.

Let $X \subseteq \mathbb{F}^{n}$. A path in $X$ made of 0 - and 1-faces is called a 1-path. A 1-path from a 0 -face $x$ to a 0 -face $y$ (with possibly $x=y$ ), is said to be elementary if its 1 -faces are all distinct. A non-trivial elementary closed path is called a $c y$ cle.

Let $X \subseteq \mathbb{F}^{n}$. We say that $X$ is connected if, for any pair of faces $(f, g)$ in $X$, there is a path in $X$ from $f$ to $g$. It is easily shown that, if $X$ is a complex, then $X$ is connected if and only if there exists an elementary path from $x$ to $y$ in $X$ whenever $x$ and $y$ are 0 -faces in $X$.

Let $X \subseteq \mathbb{F}^{n}$, and let $Y$ be a non-empty subset of $X$, we say that $Y$ is a connected component of $X$ if $Y$ is connected and if $Y$ is maximal for these two properties (i.e., if we have $Z=Y$ whenever $Y \subseteq Z \subseteq X$ and $Z$ is connected). We will sometimes write component as a shortcut for connected component. The number of components of $X$ is denoted by $|C(X)|$. Notice that $|C(\emptyset)|=0$.

\subsection{Collapse}

Let $X$ be a complex in $\mathbb{F}^{n}$ and let $f \in X$. If there exists a face $g \in \hat{f}^{*}$ such that $f$ is the only face of $X$ that strictly includes $g$, then $g$ is said to be free for $X$, and the pair $(f, g)$ is said to be a free pair for $X$. Notice that, if $(f, g)$ is a free pair for $X$, then we have necessarily $f \in X^{+}$and $\operatorname{dim}(g)=\operatorname{dim}(f)-1$.

Let $X$ be a complex, and let $(f, g)$ be a free pair for $X$. Let $m=\operatorname{dim}(f)$. The complex $X \backslash\{f, g\}$ is an elementary collapse of $X$, or an elementary m-collapse of $X$. 
Let $X, Y$ be two complexes. We say that $X$ collapses onto $Y$, and we write $X \searrow Y$, if there exists a collapse sequence from $X$ to $Y$, i.e., a sequence of complexes $\left\langle X_{i}\right\rangle_{i=0}^{\ell}(\ell \geq 0)$ such that $X_{0}=X, X_{\ell}=Y$, and $X_{i}$ is an elementary collapse of $X_{i-1}$, for each $i \in[1, \ell]$ (see Fig. 5). Let $J=\left\langle\left(f_{i}, g_{i}\right)\right\rangle_{i=1}^{\ell}$ be the sequence of pairs of faces of $X$ such that $X_{i}=X_{i-1} \backslash$ $\left\{f_{i}, g_{i}\right\}$, for any $i \in[1, \ell]$. We also call the (possibly empty) sequence $J$ a collapse sequence (from $X$ to $Y$ ). If $X$ collapses onto $Y$ and $Y$ is a complex made of a single point, we say that $X$ is collapsible.

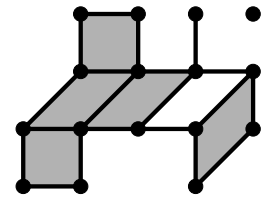

(a)

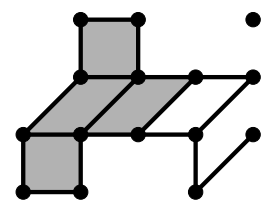

(c)

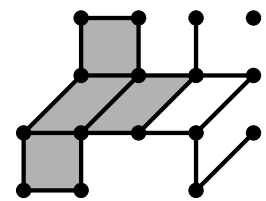

(b)

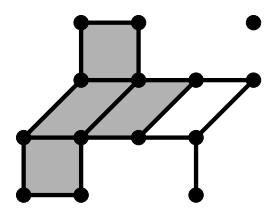

(d)
Fig. 5 (a) A complex $X$. (d) A subcomplex $Y$ of $X$. (a,b,c,d) A collapse sequence from $X$ to $Y$.

The following property is easy to prove.

Proposition 1 Let $X \leq \mathbb{F}^{n}$, let $h, k$ be two faces of $X$ and let $(f, g)$ be a free pair for $X$ such that $\{h, k\} \cap\{f, g\}=\emptyset$. If there exists a path from $h$ to $k$ in $X$, then there exists a path from $h$ to $k$ in $X \backslash\{f, g\}$.

Let $Y, X \subseteq \mathbb{F}^{n}$. We say that $X$ is an extension of $Y$ if $Y \subseteq X$ and each connected component of $X$ includes exactly one connected component of $Y$ (see [19]). The following proposition easily follows from Prop. 1.

Proposition 2 Let $Y \leq X \leq \mathbb{F}^{n}$. If $X \searrow Y$, then $X$ is an extension of $Y$. In consequence, collapse preserves the number of connected components.

Although initially formulated and proved in a framework of graphs, the next proposition and its proof can be straightforwardly adapted to cubical complexes.

Proposition 3 ([19], theorem 4) Let $Z \subseteq Y \subseteq X \subseteq \mathbb{F}^{n}$ be such that $X$ is an extension of $Z$. The subset $Y$ is an extension of $Z$ if and only if $X$ is an extension of $Y$.

Let $X \leq \mathbb{F}^{n}$, the complex that is the closure of the set of all free faces for $X$, is called the boundary of $X$, and is denoted by $B d(X)$. We denote by $B d_{1}(X)$ the complex that is the closure of the set of all free 1-faces for $X$ (see Fig. 6). Of course, we have $B d_{1}(X) \leq B d(X)$.

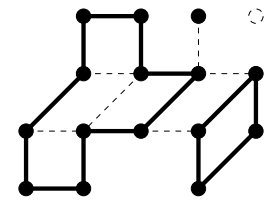

(a)

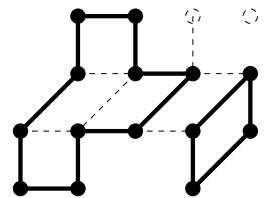

(b)
Fig. 6 (a) $B d(X)$, where $X$ is the complex of Fig. 5a. (b) $B d_{1}(X)$.

Proposition 4 Let $Y \leq X \leq \mathbb{F}^{n}$, let $\alpha$ be a set of facets of $X$ that are not in $Y$, i.e., $\alpha \subseteq X^{+} \backslash Y$. If $B d\left(\alpha^{-}\right) \subseteq Y$, then $X$ does not collapse onto $Y$.

Proof The proposition trivially holds when $X=Y \cup \alpha^{-}$. Suppose that $(f, g)$ is a free pair for $X$ outside $Y$. We see that $g$ cannot be in $\alpha$ because it is not a facet, and that $f$ cannot be in $\alpha$, otherwise $(f, g)$ would also be a free pair for $\alpha^{-}$ outside $Y$ (a contradiction with $B d\left(\alpha^{-}\right) \subseteq Y$ ). By induction we deduce that any complex $Z$ such that $Y \leq Z$ and $X \searrow Z$ includes $Y \cup \alpha^{-}$, hence the proposition.

The following property can be easily derived from Prop. 4 .

Proposition 5 Let $Y \leq X \leq \mathbb{F}^{n}$ be such that $\operatorname{dim}(X \backslash Y)=1$. If there exists a cycle in $X$ that contains at least one 1-facet of $X$ which is outside $Y$, then $X$ does not collapse onto $Y$.

Proposition 6 Let $Z \leq X \leq \mathbb{F}^{n}$ be two complexes such that $X \searrow Z$. Let $J=\left\langle\left(f_{i}, g_{i}\right)\right\rangle_{i=1}^{\ell}$ be a collapse sequence from $X$ to $Z$. Suppose that there exists $Y \leq X$ such that $Z \leq Y$ and for any $i \in[1, \ell]$, either $\left\{f_{i}, g_{i}\right\} \subseteq Y$ or $\left\{f_{i}, g_{i}\right\} \subseteq X \backslash Y$. Then, $X \searrow Y$ and $Y \searrow Z$.

Proof Let $k \in[2, \ell]$ be such that $f_{k}, g_{k} \notin Y$ and $f_{k-1}, g_{k-1} \in$ $Y$, if such an integer exists. Since $g_{k} \nsubseteq f_{k-1}$ (otherwise $g_{k} \in$ $\left.Y^{-}=Y\right),\left(f_{k}, g_{k}\right)$ is a free pair for $X \backslash\left\{f_{i}, g_{i}\right\}_{i=1}^{k-2}$ and we can swap the two pairs in $J$, still getting a collapse sequence. By repeating this procedure, we can build a collapse sequence from $X$ to $Z$ where the first $m$ pairs $(m \in[0, \ell])$ are not in $Y$ and the last $\ell-m$ pairs are in $Y$. It can easily be seen that the first $m$ pairs (resp. the last $\ell-m$ pairs) of this new sequence constitute a collapse sequence from $X$ to $Y$ (resp. from $Y$ to $Z$ ). $\square$

Let $J=\left\langle\left(f_{i}, g_{i}\right)\right\rangle_{i=1}^{\ell}$ be a collapse sequence. This collapse sequence is said to be decreasing if for any $i \in[1, \ell-1]$, we have $\operatorname{dim}\left(f_{i}\right) \geq \operatorname{dim}\left(f_{i+1}\right)$. Prop. 7 may be proved in a similar manner as Prop. 6.

Proposition 7 ([20]) Let $Y \leq X \leq \mathbb{F}^{n}$. If $X$ collapses onto $Y$, then there exists a decreasing collapse sequence from $X$ to $Y$.

Let $X, Y$ be two complexes. Let $Z$ be such that $X \cap Y \leq$ $Z \leq Y$, and let $f, g \in Z \backslash X$. The pair $(f, g)$ is a free pair for $X \cup Z$ if and only if $(f, g)$ is a free pair for $Z$. Thus, by induction, we have the following property. 
Proposition 8 ([15]) Let $X, Y \leq \mathbb{F}^{n}$. The complex $X \cup Y$ collapses onto $X$ if and only if the complex $Y$ collapses onto $X \cap Y$.

\subsection{Simple sets}

The operation of detachment allows us to remove a subcomplex from a complex while guaranteeing that the result is still a complex (see Fig. 7).

Definition 1 ([15]) Let $Y \leq X \leq \mathbb{F}^{n}$. We set $X \otimes Y=$ $\left(X^{+} \backslash Y^{+}\right)^{-}$. The set $X \otimes Y$ is a complex that is called the detachment of $Y$ from $X$.

Intuitively a cell $\hat{f}$ or a subcomplex $Y$ of a complex $X$ is simple if its removal from $X$ "does not modify the topology of $X$ ". Let us now recall a definition of simplicity [15] based on the collapse operation, which can be seen as a discrete counterpart of the one given by T.Y. Kong [14].

Definition 2 ([15]) Let $Y \leq X \leq \mathbb{F}^{n}$. We say that $Y$ is simple for $X$ if $X$ collapses onto $X \otimes Y$. If $\hat{f}$ is a simple cell, we will also say that $f$ is simple. (a)

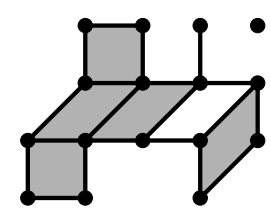

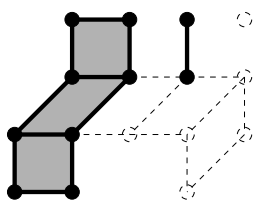

(b)

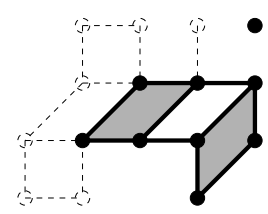

(c)

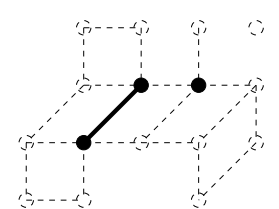

(d)

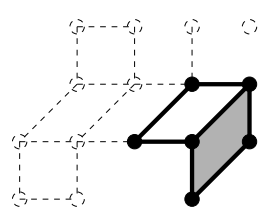

(e)
Fig. 7 (a) A complex $X$. (b) A subcomplex $Y$ of $X$ that is simple for $X$. (c) The detachment of $Y$ from $X$. (d) The attachment of $Y$ to $X$. (e) A subcomplex $Z$ of $X$ that is not simple for $X$.

The following remarks highlight some links between this framework and digital topology:

(a) If $P$ is any finite set of faces in $\mathbb{F}^{n}$ such that no element of $P$ is contained in another element of $P$, then one can define a simple subset of $P$ to be a subset $S$ of $P$ such that $S^{-}$is simple for $P^{-}$(or, equivalently, $P^{-}$collapses to $(P \backslash S)^{-}$.

(b) If $P$ is any finite set of 2 -faces in $\mathbb{F}^{2}$ (i.e., a "finite set of pixels"), then a subset of $P$ would be simple in the sense of (a) if and only if that subset is strongly deletable (see introduction). (c) There are some papers on digital topology in which the concept of a simple subset is defined in a different way that is inequivalent to (a) when $P^{-}$is an arbitrary cubical complex; a fairly recent example is [21].

The notion of attachment, as introduced by T.Y. Kong [22,14], leads to a local characterisation of simple sets (Prop. 9).

Let $Y \leq X \leq \mathbb{F}^{n}$. The attachment of $Y$ for $X$ is the complex defined by $\operatorname{Att}(Y, X)=Y \cap(X \otimes Y)$ (see Fig. 7). Remark that any facet $f$ of $X$ such that $\operatorname{Att}(\hat{f}, X) \neq \hat{f}^{*}$ includes a free face for $X$. $Y=X$.

Prop. 9 is a special case of Prop. 8 , as we have $(X \odot Y) \cup$

Proposition 9 ([15]) Let $Y \leq X \leq \mathbb{F}^{n}$. The complex $Y$ is simple for $X$ if and only if $Y$ collapses onto $\operatorname{Att}(Y, X)$.

For example in Fig. 7, it may be easily checked, both from the definition and using Prop. 9, that $Y$ is simple for $X$.

Remark 1 If $Y=\emptyset$, or if $Y \leq X$ contains no facet of $X$, then $Y$ is obviously a simple set for $X$, as we have $X \otimes Y=X$. More generally, it can be proved [17] that the detachment of a subcomplex $Y$ from $X$ is equal to the detachment of the maximal principal subcomplex $Z$ of $X$ included in $Y$. Without loss of generality, the study of the simple sets $Y$ of a complex $X$ can then be restricted to those verifying $Y \sqsubseteq X$ and $Y \neq \emptyset$. From now on, we will always implicitly consider that a simple set verifies these hypotheses.

\section{Confluence properties in cubical complexes}

Consider three complexes $A, B, C$. If $A$ collapses onto $C$ and $A$ collapses onto $B$, then we know that $A, B$ and $C$ "have the same topology". If furthermore we have $C \leq B \leq A$, it is tempting to conjecture that $B$ collapses onto $C$. We call this a confluence property. For example, this property implies that any complex in $\mathbb{F}^{2}$ obtained by a collapse sequence from a full rectangle indeed collapses onto a point.

Quite surprisingly, such a property does not hold in $\mathbb{F}^{3}$ (more generally in $\mathbb{F}^{n}, n \geq 3$ ), and this fact constitutes one of the principal difficulties when dealing with certain global topological properties. A classical counter-example to this assertion is Bing's house ([23], see also [7,5]). In Fig. 8, we see a classical representation of Bing's house. The house has two rooms separated by a floor; one can enter the lower room of the house by the chimney passing through the upper room, and vice-versa. A realisation of Bing's house as a 2complex can be obtained by collapse from a full cuboid, and has no free face: it is thus a counter-example for the above conjecture, with $A$ : a cuboid, $B$ : Bing's house, and $C$ : a point in $B$. 


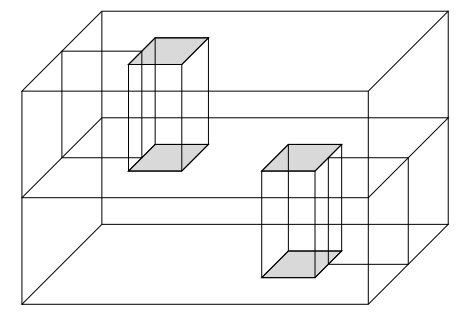

Fig. 8 Bing's house with two rooms (classical representation). The four rectangles in light grey are not part of the house, thus the lower room can be reached through the upper chimney, and vice-versa.

As we will show in this article, in the two-dimensional discrete plane $\mathbb{F}^{2}$ and more generally in the class of discrete spaces called pseudomanifolds, a confluence property indeed holds (Th. 2).

We first establish a confluence property that is essentially 1-dimensional, a step for proving more general confluence properties.

A tree is a graph that is collapsible. It may be easily proved that a graph is a tree if and only if it is connected and does not contain any cycle (see [18]).

Let $X \leq \mathbb{F}^{n}$ be a complex. The set of all $i$-faces of $X$, with $i \in[0, n]$, is denoted by $F_{i}(X)$. We denote by $\left|F_{i}(X)\right|$ the number of $i$-faces of $X, i \in[0, n]$. The Euler characteristic of $X$, written $\chi(X)$, is defined by $\chi(X)=\sum_{i=0}^{n}(-1)^{i}\left|F_{i}(X)\right|$. The Euler characteristic is a well-known topological invariant; it can be easily seen that collapsing preserves it.

The following property generalises a classical characterisation of trees: a graph $X$ is a tree if and only if $X$ is connected and $\chi(X)=1$.

Proposition 10 Let $X, Y$ be such that $Y \leq X \leq \mathbb{F}^{n}$, and $\operatorname{dim}(X \backslash Y) \leq 1$. Then, $X$ collapses onto $Y$ if and only if $X$ is an extension of $Y$ and $\chi(Y)=\chi(X)$.

From Prop. 10 (which is proved in the appendix), we can establish the following property, from which derives Prop. 12.

Proposition 11 Let $C \leq B \leq A \leq \mathbb{F}^{n}$ be such that $\operatorname{dim}(A \backslash$ $C) \leq 2$ and $A$ collapses onto $C$. Then $B$ collapses onto $C$ if and only if $B$ is an extension of $C$ and $\chi(B)=\chi(C)$.

Proof The "only if" part of the proof is straightforward, let us prove the "if" part. Since $A$ collapses onto $C$, we know that there exists a collapse sequence $S=\left\langle\left(f_{i}, g_{i}\right)\right\rangle_{i=1}^{\ell}$ from $A$ to $C$. As $B \leq A$ and $\operatorname{dim}(A \backslash C) \leq 2$, no 2-face in $B \backslash C$ is a $g_{i}$, and so each 2-face in $B \backslash C$ is an $f_{i}$.

We claim that the subsequence of $S$ that consists of the pairs $\left(f_{i}, g_{i}\right)$ for which $f_{i}$ is a 2-face in $B \backslash C$ is a collapse sequence from $B$ to a complex $B^{\prime}$ such that $C \leq B^{\prime}$ and $\operatorname{dim}\left(B^{\prime} \backslash C\right) \leq 1$. To justify this claim, consider the first index $t$ such that $f_{t}$ is a 2-face in $B \backslash C$, if any such face exists (otherwise our claim holds with $B^{\prime}=B$ ). Let $A_{1}=A \backslash\left\{f_{i}, g_{i}\right\}_{i=1}^{t-1}$.
We know that $\left(f_{t}, g_{t}\right)$ is free for $A_{1}$, that is, $f_{t}$ is the only face of $A_{1}$ that strictly includes $g_{t}$. Since $\operatorname{dim} B \backslash A_{1} \leq 1$ (by definition of $f_{t}$ ) and $f_{t} \in B$, we see that $f_{t}$ is also the only face of $B$ that strictly includes $g_{t}$, i.e., $\left(f_{t}, g_{t}\right)$ is free for $B$. Let $B_{1}=B \backslash\left\{f_{t}, g_{t}\right\}$, we have $C \leq B_{1} \leq B \leq A$ and $B$ collapses onto $B_{1}$. Still considering the same collapse sequence $S$, and substituting $B_{1}$ to $B$, we can repeat the same argument. Eventually, we obtain a collapse sequence $\left\langle B, B_{1}, \ldots, B_{k}=B^{\prime}\right\rangle$ such that $C \leq B^{\prime}$ and $\operatorname{dim}\left(B^{\prime} \backslash C\right) \leq 1$.

Now, suppose that $B$ is an extension of $C$ and $\chi(C)=$ $\chi(B)$. As $B$ collapses to $B^{\prime}$, we have that $B$ is an extension of $B^{\prime}$ (Prop. 2), and by Prop. 3 we deduce that $B^{\prime}$ is an extension of $C$. Furthermore, $\chi\left(B^{\prime}\right)=\chi(B)=\chi(C)$. So Prop. 10 implies that $B^{\prime}$ collapses onto $C$, hence $B$ collapses onto $C$. $\square$

Prop. 12 is an immediate corollary of Prop. 11.

Proposition 12 (Downstream confluence) Let $A, B, C$ be such that $C \leq B \leq A \leq \mathbb{F}^{n}$ and such that $\operatorname{dim}(A \backslash C) \leq 2$. If A collapses onto $C$ and $A$ collapses onto $B$, then $B$ collapses onto $C$.

From Props. 3, 10, and the fact that collapse preserves the Euler characteristic, we also derive straightforwardly the following proposition.

Proposition 13 (1-D Upstream confluence) Let $A, B, C$ be such that $C \leq B \leq A \leq \mathbb{F}^{n}$ and such that $\operatorname{dim}(A \backslash B) \leq 1$. If $A$ collapses onto $C$ and $B$ collapses onto $C$, then $A$ collapses onto $B$.

The following property of graphs, a necessary and sufficient condition which accounts for both downtream and upstream confluences, derives immediately from Props. 12 and 13.

Proposition 14 (Confluence in graphs) Let $A$ be a graph and let $B, C$ be such that $C \leq B \leq A$ and $A$ collapses onto $C$. Then, $B$ collapses onto $C$ if and only if $A$ collapses onto $B$.

\section{Two-dimensional pseudomanifolds}

Intuitively, a (2-D) manifold ${ }^{2}$ is a 2-D (finite or infinite) space which is locally "like" the 2-D Euclidean space (spheres and tori are, for instance, manifolds).

The notion of (2-D) pseudomanifold is less restrictive since it authorises several pieces of surface to be adjacent in a singular point (as two cones sharing the same apex, for instance). Note that any manifold is a pseudomanifold, but the converse is not true. Some examples of pseudomanifolds are provided in Fig. 9.

\footnotetext{
${ }^{2}$ In this article, the notions of manifold and pseudomanifold will implicitly refer to objects without boundary. Formal definitions of these notions may be found e.g. in [24].
} 


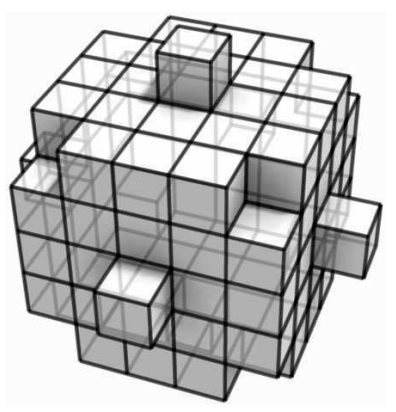

(a)

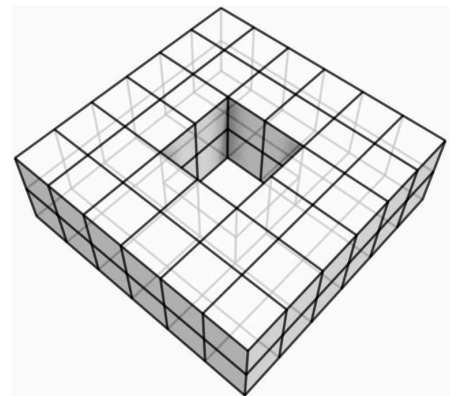

(b)

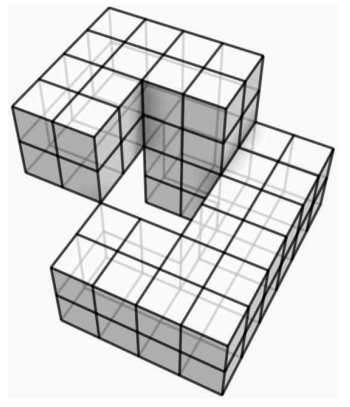

(c)
Fig. 9 2-D pseudomanifolds. (a) A topological sphere. (b) A topological torus. (c) A pinched torus. (a) and (b) are manifolds (thus also pseudomanifolds), (c) is a pseudomanifold but not a manifold.

In the framework of cubical complexes, a 2-D pseudomanifold can be defined as follows. We denote by $\mathbb{F}_{2}^{n}$ the set composed of all $m$-faces of $\mathbb{Z}^{n}$, with $m \in[0,2]$. We say that $\pi$ is a 2-path (in $X$ ) if $\pi$ is a path in $X$ composed of 1- and 2 -faces.

Definition 3 Let $M \subseteq \mathbb{F}_{2}^{n}$ be such that $\operatorname{dim}(M)=2$. We say that $M$ is a (2-D) pseudomanifold if the following four conditions hold:

(i) for any $f \in M$, we have $\hat{f} \subseteq M$;

(ii) $M$ is pure;

(iii) for any pair of 2-faces $(f, g)$ in $M$, there is a 2-path in $M$ from $f$ to $g$;

(iv) any 1-face of $M$ is included in exactly two 2-faces of $M$.

Notice that, in particular, $\mathbb{F}_{2}^{2}=\mathbb{F}^{2}$ (namely the discrete plane) is a pseudomanifold. Notice also that, if $M$ is a finite pseudomanifold, then $M$ is a pure 2-complex that cannot be collapsed, since $M$ has no free face by definition.

In the sequel, we focus on complexes that are strict subsets of a pseudomanifold, as illustrated in Fig. 10.

Proposition 15 Let $M \subseteq \mathbb{F}_{2}^{n}$ be a pseudomanifold, and let $X \leq M$. Then, $B d\left(B d_{1}(X)\right)=B d(B d(X))=\emptyset$.

Proof It is plain that $B d\left(B d_{1}(X)\right)=B d(B d(X))$. Let $p$ be a point in $B d_{1}(X)$, and let $E$ (resp. $S$ ) be the set of edges (resp. squares) of $X$ including $p$. We write $d_{k}$ to denote the

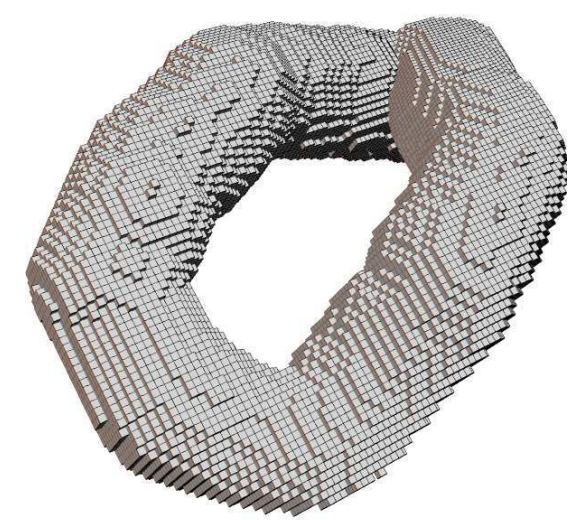

(a)

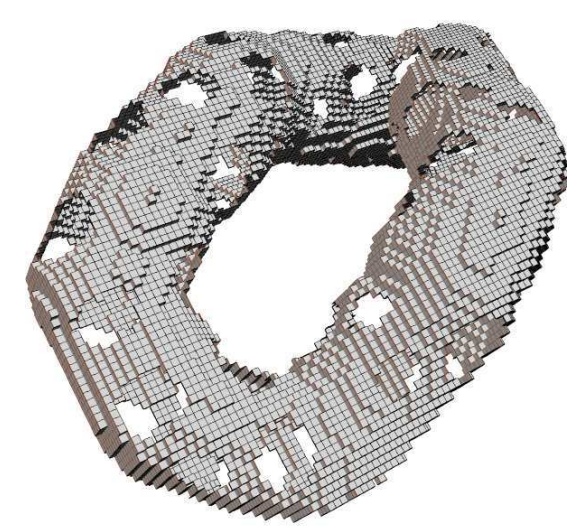

(b)

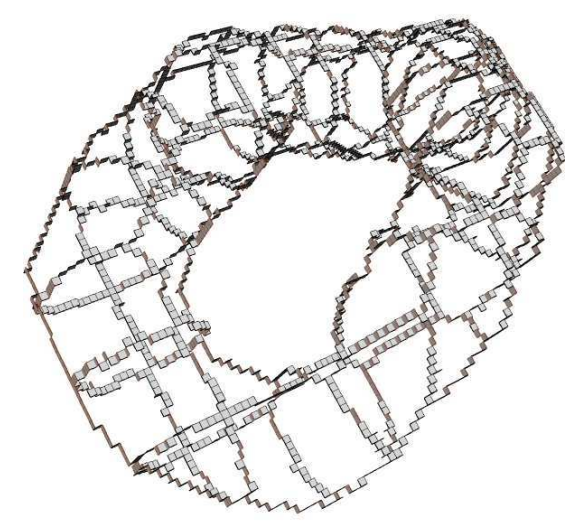

(c)

Fig. 10 (a) A 2-D pseudomanifold $M$, having the topology of a (hollow) torus. (b) A subcomplex $X$ of $M$ (some disks have been removed). (c) Another subcomplex $Y$ of $M$, such that $X$ collapses onto $Y$.

number of edges of $E$ included in exactly $k$ squares of $S$. As $M$ is a pseudomanifold, $d_{k}=0$ for all $k>2$. Of course, each square of $S$ includes exactly two edges of $E$. Thus, $2|S|=0 d_{0}+1 d_{1}+2 d_{2}$, implying that $d_{1}$ is even, hence $p \notin$ $B d\left(B d_{1}(X)\right)$. Since this holds for any $p \in B d_{1}(X)$, we have $B d\left(B d_{1}(X)\right)=\emptyset$. 
Proposition 16 Let $M \subseteq \mathbb{F}_{2}^{n}$ be a pseudomanifold, let $B \leq$ $M$ such that $\operatorname{dim}(B)=2$ and $B \neq M$, let $f$ be a 2 -face of $B$, and let $g$ be a 2-face in $M \backslash B$. If $\pi$ is a 2-path from $f$ to $g$ in $M$, then $\pi$ necessarily contains a 1-face of $B d(B)$.

Proof Let $h$ be the first 2-face of $\pi$ that is not in $B$, let $k$ be the last 2-face of $\pi$ before $h$ (thus $k$ is in $B$ ), and let $e=k \cap h$ be the 1 -face of $\pi$ between $k$ and $h$. Since $M$ is a pseudomanifold, $e$ is included in exactly two 2-faces of $M$ and in exactly one 2 -face of $B$, that is, $e \in B d_{1}(B)$. $\square$

Prop. 17 follows easily from Prop. 16.

Proposition 17 Let $M \subseteq \mathbb{F}_{2}^{n}$ be a pseudomanifold, let $B \leq$ M. If $\operatorname{dim}(B)=2$ and $B \neq M$, then there exists at least one pair $(f, g)$ that is free for $B$, with $\operatorname{dim}(f)=2$.

\section{A confluence property in 2-D pseudomanifolds}

Recall that $X$ collapses onto $Y$ if and only if $X$ is an extension of $Y$ and $\chi(Y)=\chi(X)$, provided that $Y \leq X \leq \mathbb{F}^{n}$ and $\operatorname{dim}(X \backslash Y) \leq 1$ (Prop. 10). It is tempting to try to generalise this property to the case where $X$ and $Y$ are any subcomplexes of a pseudomanifold, for confluence properties would immediately follow from such a result. But in fact, the backward implication of Prop. 10 does not hold in the general case (that is, when $\operatorname{dim}(X \backslash Y)$ is not constrained), even if $X$ and $Y$ are complexes that are subsets of a pseudomanifold.

To get a counter-example, let us consider as $X$, the complex $Z$ of Fig. 7(e) (which can be embedded in a pseudomanifold, for instance the boundary of a cube), and let $Y=$ $B d(X)$ (a topological circle). It is plain that $X$ is an extension of $Y$, while $\chi(X)=\chi(Y)=0$. However, by construction, $X$ has no free face outside $Y$, thus $X$ does not collapse onto $Y$.

Nevertheless, we can prove the following property, that will be used in the next section.

Proposition 18 Let $M \subseteq \mathbb{F}_{2}^{n}$ be a pseudomanifold, and let $X \leq M, X \neq M$. The complex $X$ is collapsible if and only if $|C(X)|=\chi(X)=1$.

Proof The forward implication is immediate, let us prove the converse. Suppose that $|C(X)|=\chi(X)=1$. If $\operatorname{dim}(X) \leq 1$ (i.e., if $\left|F_{2}(X)\right|=0$ ), then by Prop. 10 we deduce that $X$ is collapsible.

Suppose now that $\operatorname{dim}(X)=2$. Since $X \neq M$, by Prop. 17 we know that $X$ has at least one free pair, and the result follows by induction on $\left|F_{2}(X)\right|$.

We already know that the downstream confluence property holds in 2-D pseudomanifolds, as a particular case of Prop. 12. To have a general confluence property, similar to Prop. 14 in graphs, we need to prove the upstream confluence.
Proposition 19 (Upstream confluence) Let $M \subseteq \mathbb{F}_{2}^{n}$ be a pseudomanifold, and let $C \leq B \leq A \leq M$. If $A$ collapses onto $C$ and $B$ collapses onto $C$, then $A$ collapses onto $B$.

Proof If $\left|F_{2}(A)\right|=0$ then by Prop. 13, $A \searrow B$. Suppose that $\left|F_{2}(A)\right|>0$ and that the proposition holds when $A$ is replaced with any $A^{\prime}$ such that $\left|F_{2}\left(A^{\prime}\right)\right|<\left|F_{2}(A)\right|$. Consider the set $\alpha$ of 1 -faces that are free for $A$ and not in $C$, i.e., $\alpha=F_{1}(B d(A) \backslash C)$. If $\alpha=\emptyset$, then the hypothesis $A \searrow C$ implies that $\left|F_{2}(A)\right|=\left|F_{2}(C)\right|=\left|F_{2}(B)\right|$, and the result follows from Prop. 13. We now suppose that $\alpha \neq \emptyset$. By Prop. 15, no face in $B d(A)$ is free for $B d(A)$, hence no face in $\alpha^{-}$is free for $\alpha^{-} \cup C$. Thus, the faces in $\alpha$ cannot all be facets of $B$, for otherwise, by Prop. $4, B$ could not collapse onto $C$. From this, we deduce that there exists a 1-face $g$ in $\alpha$ such that either $g \in B d(B)$ or $g \notin B$. Let $f$ be the 2-face of $A$ that includes $g$.

Case 1: $g \in B d(B)$. Thus, $(f, g)$ is a free pair for both $A$ and $B$. Let $A^{\prime}=A \backslash\{f, g\}$ and $B^{\prime}=B \backslash\{f, g\}$. We have $C \leq B^{\prime} \leq A^{\prime}, A^{\prime} \searrow C$ (by Prop. 12) and $B^{\prime} \searrow C$ (also by Prop. 12), thus by the recurrence hypothesis $A^{\prime} \searrow B^{\prime}$. Since $A^{\prime}$ collapses onto $B^{\prime}=B \cap A^{\prime}$, it follows from Prop. 8 that $B \cup A^{\prime}=A$ collapses onto $B$.

Case 2: $g \notin B$. Thus, $(f, g)$ is a free pair for $A$ that is not in $B$, let $A^{\prime}=A \backslash\{f, g\}$. We have $C \leq B \leq A^{\prime}, A^{\prime} \searrow C$ (by Prop. 12) and $B \searrow C$, thus by the recurrence hypothesis $A^{\prime} \searrow B$ hence $A \searrow B$. $\square$

The following theorem follows from Props. 12 and 19.

Theorem 2 (Confluence) Let $M \subseteq \mathbb{F}_{2}^{n}$ be a pseudomanifold, and let $C \leq B \leq A \leq M$ be such that $A$ collapses onto $C$. Then, $A$ collapses onto $B$ if and only if $B$ collapses onto $C$.

\section{Minimal simple sets in pseudomanifolds}

Informally, a minimal simple set is a simple set which does not strictly include any other simple set. In $[17,7,25]$ the notion of minimal simple set is studied and several examples of non-trivial minimal simple sets in $\mathbb{F}^{3}$ and in $\mathbb{F}_{2}^{3}$ are given.

In this section, we first establish the equivalence between the notions of simple cell and minimal simple set in pseudomanifolds (Prop. 22). Then we demonstrate that, in such spaces, any simple set can be fully detached by iterative detachment of simple cells, in any possible order (Th. 3).

Definition 4 ([17]) Let $X \leq \mathbb{F}^{n}$ and $S \sqsubseteq X$. The subcomplex $S$ is a minimal simple set (for $X$ ) if $S$ is a nonempty simple set for $X$ and $S$ is minimal with respect to the relation $\sqsubseteq$ (i.e. $Z=S$ whenever $\emptyset \neq Z \sqsubseteq S$ and $Z$ is a simple set for $X$ ).

Proposition 20 (See also [17]) Let $S \sqsubseteq X \preceq \mathbb{F}_{2}^{n}$ such that $S$ is a minimal simple set for $X$. Then, $S$ is connected. 
Proof Let $S_{1}$ be a connected component of $S$. Remark that $S_{1} \neq \emptyset$ and $S_{1} \sqsubseteq S$. Let $J=\left\langle\left(f_{i}, g_{i}\right)\right\rangle_{i=1}^{\ell}$ be a collapse sequence from $X$ to $X \otimes S$. Any pair $\left(f_{i}, g_{i}\right)$ is either in $X \otimes S_{1}$ or in $S_{1} \backslash \operatorname{Att}\left(S_{1}, X\right)=X \backslash\left(X \odot S_{1}\right)$, thus by Prop. 6, $X \searrow X \odot S_{1}$. Hence $S_{1}$ is a simple set for $X$, and the minimality of $S$ then implies that $S=S_{1}$.

Proposition 21 Let $X \leq \mathbb{F}^{n}$ be a connected 2-complex, let $S \sqsubseteq X$ be a simple subcomplex of $X$, and let $f$ be a facet of $S$ such that $A t t(\hat{f}, X)$ is not empty and not connected. Then, $X \otimes \hat{f}$ is an extension of $\operatorname{Att}(\hat{f}, X)$.

Proof Let us assume that $\operatorname{dim}(f)=2$ (the case where $\operatorname{dim}(f)=1$ is similar and simpler). Let us write $A=\operatorname{Att}(\hat{f}, X)$ and $B=X \otimes \hat{f}$. All the different possible configurations for $A$, up to symmetries and rotations, are shown below. The elements of $A$ are depicted by black vertices and bold edges.

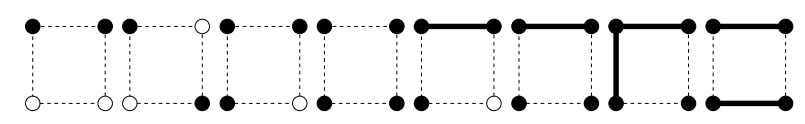

Consider two distinct connected components $C, D$ in $A$. Choose a 1-face $g$ in $\hat{f}^{*} \backslash A$ such that there exists an elementary path $\pi$ from a 0 -face $c$ of $C$ to a 0 -face $d$ of $D$ in $\hat{f}^{*}$ that does not contain $g$ nor any 1 -face of $A$. One can verify by inspection that such a choice is possible in all eight configurations above, whatever the considered $C, D$. The pair $(f, g)$ is obviously free for $X$.

Let $X^{\prime}=X \backslash\{f, g\}$. Since $X \searrow X \otimes S$ and $X \searrow X^{\prime}$, by Prop. 12 we have $X^{\prime} \searrow X \otimes S$, and by Prop. 7 there exists a sequence of 2-collapse operations from $X^{\prime}$ to a complex $Z$ such that $X \otimes S \leq Z$ and $Z$ has no 2-face outside $X \otimes S$. By Prop. 7 we also deduce $Z \searrow X \otimes S$, and observe that $\pi$ is in $Z$ since any 1 -face of $\pi$ is not in any 2-face of $X^{\prime}$.

Suppose that $C$ and $D$ are in a same connected component of $B=X \otimes \hat{f}$. Then, by Prop. 1 there exists an elementary path $\sigma$ from $d$ to $c$ in $Z \otimes \hat{f}$. It can be seen that, by construction, $\sigma$ cannot contain any 1 -face of $\pi$, and that any 1 -face of $\pi$ cannot be in $X \otimes S$. Thus the concatenation of $\sigma$ and $\pi$ forms a cycle in $Z$ having at least a 1-face outside $X \odot S$, contradicting Prop. 5 and the fact that $Z \searrow X \otimes S$. We conclude that any two distinct components of $A$ are in distinct components of $B$. Since furthermore each component of $A$ is included in a component of $B$, we get the result.

Proposition 22 Let $M \subseteq \mathbb{F}_{2}^{n}$ be a pseudomanifold, and let $S \sqsubseteq X \preceq M$ such that $S$ is a minimal simple set for $X$. Then, $S$ is necessarily a 1-cell or a 2-cell.

Proof Suppose that $S$ is not just one cell. Then, each facet of $S$ must be non-simple for $X$. However, since $S$ is simple, no facet $f$ of $S$ is such that $\operatorname{Att}(\hat{f}, X)=\emptyset$. If $S$ contains a 1 -facet, then let $f$ be such a facet. If $S$ is a pure 2-complex, then at least one 2-face of $S$ must include a free face for $X$, since $X$ collapses onto $X \odot S$, and we assume that $f$ is such a 2-face. Let $A=\operatorname{Att}(\hat{f}, X)$. In both cases $(\operatorname{dim}(f)=1$ or $\operatorname{dim}(f)=2$ ), we know that $A$ is disconnected. We claim $S$ has a nonempty subcomplex that is simple for $X$ but does not contain $f$. This claim contradicts the minimality of $S$, so if we can justify it then the proof will be complete. In justifying this claim, we suppose that $\operatorname{dim}(f)=2$ (the case where $\operatorname{dim}(f)=1$ is similar and simpler).

From Prop. 20, $S$ is connected and from Props. 9 and 2, $\operatorname{Att}(S, X)$ is connected. Without loss of generality, we assume that $X$ is connected (otherwise we replace $X$ by the component of $X$ that includes $S$ ). By Prop. 21, each component of $X \otimes \hat{f}$ includes exactly one component of $A$. Let $X_{1}$ be the component of $X \otimes \hat{f}$ that includes $\operatorname{Att}(S, X)$ (and thus also $X \otimes S$ ), and let $A_{1}$ be the component of $A$ that is in $X_{1}$. Let $g$ and $h$ be the two 1 -faces of $\hat{f}^{*} \backslash A$ that each include a 0 -face of $A_{1}$. Obviously $(f, g)$ is a free pair for $X$; let $X^{\prime}=X \backslash\{f, g\}$. Then $h$ is a facet of $X^{\prime}$. We have $X \searrow X^{\prime}$ and $X \searrow X \otimes S$, so by Prop. 12 we deduce $X^{\prime} \searrow X \otimes S$.

Let $J=\left\langle\left(f_{i}, g_{i}\right)\right\rangle_{i=1}^{\ell}$ be a collapse sequence from $X^{\prime}$ to $X \otimes S$. Let $t \in[1, \ell]$ be such that $f_{t}=h$. It can be seen that $g_{t} \notin X_{1}$ (otherwise the result of the collapse operation would be disconnected, for by construction any path in $X^{\prime}$ from $X \otimes S$ to the remaining face in $h$ would contain $h$ ), and of course $f_{t} \notin X_{1}$. Furthermore, any other pair of $J$ is either in $X_{1}$ or in $X^{\prime} \backslash X_{1}$, since the only facet of $X^{\prime} \backslash X_{1}$ that includes a face of $X_{1}$ is $f_{t}$. Thus by Prop. 6, $X^{\prime} \searrow X_{1}$, hence $X \searrow X_{1}$.

It is plain that $\hat{f} \searrow A_{1}$, thus by Prop. 8 we have $X_{1} \cup \hat{f} \searrow X_{1}$; and since $X \searrow X_{1}$, by Prop. 19 we deduce that $X \searrow X_{1} \cup \hat{f}$, i.e., $X \ominus\left(X_{1} \cup \hat{f}\right)$ is a simple set for $X$. This justifies our claim and contradicts the minimality of $S$, since it follows from the definition of $X_{1}$ that $X \ominus\left(X_{1} \cup \hat{f}\right) \sqsubseteq S$.

From Props. 22 and 12, we derive straightforwardly our main theorem.

Theorem 3 Let $M \subseteq \mathbb{F}_{2}^{n}$ be a pseudomanifold, and let $\emptyset \neq$ $S \sqsubseteq X \preceq M$ such that $S$ is a simple set for $X$. Then:

(i) there is a facet of $X$ in $S$ which is simple for $X$; and

(ii) for any cell $\hat{f}$ in $S$ which is simple for $X, S \otimes \hat{f}$ is a simple set for $X \otimes \hat{f}$.

To illustrate this property, consider the 2-complexes $M$, $X$ and $Y$ displayed in Fig. 10. If we know that $X$ collapses onto $Y$ (i.e., $X \odot Y$ is a simple set for $X$ ), then Th. 3 tells us that we can obtain $Y$ from $X$ by sequentially removing simple cells from $X \odot Y$, in any arbitrary order.

It has to be noticed, that the pseudomanifold hypothesis is essentially used to prove Prop. 19. All other intermediate steps do not directly need this hypothesis. Then, a natural question follows: does Prop. 19 (and also Th. 3 by consequence) extends to the family of unrestricted 2-complexes in $\mathbb{F}^{n}$ ? 
The answer to this question is negative, for there exists some minimal simple sets in this family which are not single cells, and there exists some non-trivial collapsible 2complexes that do not contain any simple facet. Examples of such configurations are given in [25]. The same counterexamples forbid to generalize Prop. 19 to the case where $\operatorname{dim}(A \backslash C) \leq 2$, like in Prop. 12, instead of assuming $A, B, C$ to be subcomplexes of a pseudomanifold.

A proposition derived from Prop. 18 and Th. 3, presented below (see also [26]), will serve us to establish a new property relative to parallel thinning (Th. 5, Sec. 7).

Proposition 23 Let $M \subseteq \mathbb{F}_{2}^{n}$ be a pseudomanifold, and let $X \leq M, X \neq M$. If $|C(X)|=\chi(X)=1$ and $X$ has no simple facet, then $X$ is a single cell.

Proof Suppose that $|C(X)|=\chi(X)=1$, that $X$ has no simple facet, and that $X$ is not a single cell. By Prop. 18 we know that $X$ is collapsible, i.e., there is a point (i.e., a 0 -face) $g$ in $X$ such that $X \searrow \hat{g}$; let $f$ be a facet of $X$ that includes $g$. We have $X \searrow \hat{g}$ and $\hat{f} \searrow \hat{g}$, thus by Th. 2 we deduce $X \searrow \hat{f}$, i.e., the set $S=X \otimes \hat{f}$ is a simple set for $X$. Since $X \neq X \otimes S=\hat{f}$, by Th. 3 we know that $S$ contains a facet of $X$ which is simple for $X$, a contradiction.

\section{Parallel thinning, critical kernels}

Th. 3 is in relation with sequential thinning algorithms. In this section, we derive from Th. 3 (more precisely, from Prop. 23) a property, Th. 5, that relates to parallel homotopic thinning. Let us first recall the framework introduced by G. Bertrand in [15] for thinning, in parallel, discrete objects with the warranty that we do not alter the topology of these objects. We focus here on the two-dimensional case, however this method is actually valid for complexes of arbitrary dimension.

The critical kernels framework is based solely on three notions, the notion of an essential face which enables to define the core of a face, and the notion of a critical face. In the sequel, $X$ represents an object and $K$ a constraint set (see Sec. 1).

Definition 5 ([15]) Let $X \leq \mathbb{F}_{2}^{n}$ and let $f \in X$. We say that $f$ is an essential face for $X$ if $f$ is precisely the intersection of all facets of $X$ which include $f$, i.e., if $f=\cap\left\{g \in X^{+} \mid f \subseteq\right.$ $g$ ). We denote by $\operatorname{Ess}(X)$ the set composed of all essential faces of $X$. If $f$ is an essential face for $X$, we say that $\hat{f}$ is an essential cell for $X$.

Observe that a facet of $X$ is necessarily an essential face for $X$, i.e., $X^{+} \subseteq \operatorname{Ess}(X)$.

Definition 6 ([15]) Let $K \leq X \leq \mathbb{F}_{2}^{n}$ and let $f \in \operatorname{Ess}(X)$. The core of $\hat{f}$ for $\langle X \mid K\rangle$ (read: $X$ constrained by $K$ ) is the complex, denoted by $\operatorname{Core}(\hat{f}, X, K)$, which is the union of all essential cells for $X$ and all cells of $K$ which are in $\hat{f}^{*}$, i.e., $\operatorname{Core}(\hat{f}, X, K)=\cup\left\{\hat{g} \mid g \in[K \cup \operatorname{Ess}(X)] \cap \hat{f}^{*}\right\}$.

Definition 7 ([15]) Let $X \leq \mathbb{F}_{2}^{n}$ and let $f \in X$. We say that $f$ and $\hat{f}$ are regular for $\langle X \mid K\rangle$ if $f \in \operatorname{Ess}(X)$ and if $\hat{f}$ collapses onto $\operatorname{Core}(\hat{f}, X, K)$. We say that $f$ and $\hat{f}$ are critical for $\langle X \mid K\rangle$ if $f \in \operatorname{Ess}(X)$ and if $f$ is not regular for $\langle X \mid K\rangle$. If $X \leq \mathbb{F}_{2}^{n}$, we set $\operatorname{Critic}(X, K)=\cup\{\hat{f} \mid f$ is critical for $\langle X \mid K\rangle\}, C r i t i c(X, K)$ is the critical kernel of $\langle X \mid K\rangle$, of simply the critical kernel of $X$ if $K=\emptyset$.

Prop. 24 follows straightforwardly from the definitions.

Proposition 24 ([16]) Let $X \leq \mathbb{F}_{2}^{n}$ and let $f \in X^{+}$. We have $\operatorname{Core}(\hat{f}, X, \emptyset)=\operatorname{Att}(\hat{f}, X)$, thus the facet $f$ is regular for $\langle X|$ $\emptyset>$ if and only if $f$ is simple for $X$.

Remark that Prop. 24 has the following consequences:

(1) No simple facet of $X$ lies in the critical kernel of $X$.

(2) If $X$ has no simple facet, then the critical kernel of $X$ is $X$ itself.

The following theorem holds for complexes of arbitrary dimension (see [15]), it may be proved in a simple manner in the 2-D case (first, we collapse regular 2-faces onto their core, then we collapse regular 1-faces onto their core). This is the basic result in this framework.

Theorem 4 ([15]) Let $K \leq Y \sqsubseteq X \leq \mathbb{F}_{2}^{n}$. The complex $X$ collapses onto its critical kernel. Furthermore, if $Y$ includes the critical kernel of $\langle X \mid K\rangle$, then $X$ collapses onto $Y$.

In [16], several parallel thinning algorithms for arbitrary 2 -dimensional cubical complexes are proposed. The fact that they all preserve topology directly follows from Th. 4 . The most fundamental thinning scheme in this framework consists of iteratively computing the critical kernel of the previous result, until stability. The output of this procedure is defined below, and illustrated in Figs. 11 and 12.

Definition 8 Let $K \leq X \leq \mathbb{F}_{2}^{n}$. We set

$$
\begin{aligned}
-\operatorname{Critic}^{0}(X, K) & =X ; \\
-\operatorname{Critic}^{i}(X, K)= & \left.\operatorname{Critic} \operatorname{Critic}^{i-1}(X, K), K\right), \text { for } i>0 ; \\
-\operatorname{Critic}^{\infty}(X, K)= & \operatorname{Critic}^{k}(X, K) \\
& \text { if } \operatorname{Critic}^{k}(X, K)=\operatorname{Critic}^{k+1}(X, K) .
\end{aligned}
$$

The complex $\operatorname{Critic}^{\infty}(X, K)$ is called the critical skeleton of $\langle X \mid K\rangle$, or simply the critical skeleton of $X$ if $K=\emptyset$.

From remarks (1) and (2) above, we can deduce that

(a) The critical skeleton has no simple facet.

(b) If $X$ has no simple facet, then the critical skeleton of $X$ is $X$ itself.

From these properties of the critical skeleton and Prop. 23, we deduce the following result.

Theorem 5 Let $M \subseteq \mathbb{F}_{2}^{n}$ be a pseudomanifold, and let $X \leq$ $M, X \neq M$. If $|C(X)|=\chi(X)=1$, then the critical skeleton of $X$ is a single cell. 


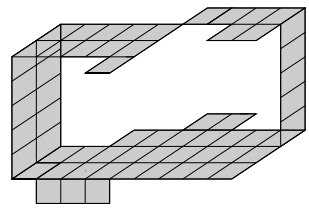

(a)

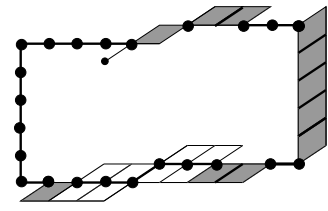

(c)

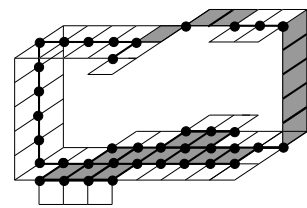

(b)

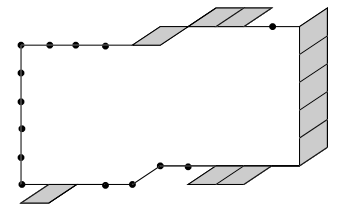

(d)
Fig. 11 (a) A 2-complex $X=X_{0}$ in $\mathbb{F}_{2}^{3}$. (b) The critical kernel $X_{1}$ of $X$ (highlighted). (c) The critical kernel $X_{2}$ of $X_{1}$ (highlighted). (d) $\operatorname{Critic}\left(X_{2}, \emptyset\right)=X_{2}=\operatorname{Critic}^{\infty}(X, \emptyset)$, the critical skeleton of $X$.

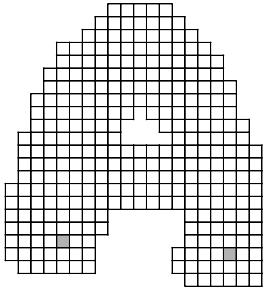

(a)

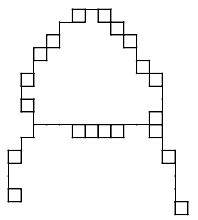

(b)
Fig. 12 (a) A complex $X \sqsubseteq \mathbb{F}^{2}$, and a constraint set $K$ (highlighted). (b) The critical skeleton of $\langle X \mid K\rangle$.

\section{Conclusion}

In this article we have established, in the case of digital 2D pseudomanifolds, a confluence property of the collapse operation (Th. 2). From this result, we have proved that in 2-D pseudomanifolds any minimal simple set is a simple cell (Prop. 22). This led us to the property stating that any simple set can be removed by iterative removal of simple cells in any order (Th. 3), and to a new property related to parallel thinning and critical kernels (Th. 5).

It is indeed possible to retrieve Ronse's theorem (Th. 1) from the results presented above, based on the equivalence between $\mathbb{Z}^{2}$ equipped with a $(8,4)$-adjacency framework and the set of pure 2-complexes in $\mathbb{F}^{2}$ [14]. For this purpose, it is necessary to prove that any subcomplex $S \sqsubseteq X$ (where $X$ is a pure 2-complex in $\mathbb{F}^{2}$ ) that is strongly deletable for $X$ is also simple for $X$ in the sense of Def. 2 (the converse also holds). This can be proved using Rosenfeld's digital Jordan curve theorem (see, e.g., Th. 7.6 of [27]).

The next step of this work will consist in studying the possible extension of these properties to 2-D complexes that are not embedded in 2-D digital spaces, but in $n$-D ones $(n \geq$ $3)$. First results will be proposed in [25].

\section{Acknowledgements}

The authors wish to thank the anonymous reviewer for introducing Prop. 11 in replacement of a less general property, and for many suggestions that significantly improved the article. tions.

They also thank John Chaussard for his help on illustra-

\section{References}

1. J.-F. Mangin, V. Frouin, I. Bloch, J. Régis, J. López-Krahe, From $3 \mathrm{D}$ magnetic resonance images to structural representations of the cortex topography using topology preserving deformations, Journal of Mathematical Imaging and Vision 5 (4) (1995) 297-318.

2. S. Faisan, N. Passat, V. Noblet, R. Chabrier, C. Meyer, Topology preserving warping of binary images: Application to atlas-based skull segmentation, in: MICCAI'08 Proceedings, Part I, Vol. 5241 of Lecture Notes in Computer Science, Springer, 2008, pp. $211-$ 218

3. N. Cornea, D. Silver, X. Yuan, R. Balasubramanian, Computing hierarchical curve-skeletons of 3D objects, The Visual Computer 21 (11) (2005) 945-955.

4. T. Y. Kong, A. Rosenfeld, Digital topology: Introduction and survey, Computer Vision, Graphics, and Image Processing 48 (3) (1989) 357-393.

5. M. Couprie, G. Bertrand, New characterizations of simple points in 2D, 3D and 4D discrete spaces, IEEE Transactions on Pattern Analysis and Machine Intelligence 31 (4) (2009) 637-648.

6. E. R. Davies, A. P. Plummer, Thinning algorithms: A critique and a new methodology, Pattern Recognition 14 (1-6) (1981) 53-63.

7. N. Passat, M. Couprie, G. Bertrand, Minimal simple pairs in the 3D cubic grid, Journal of Mathematical Imaging and Vision 32 (3) (2008) 239-249.

8. A. Rosenfeld, Connectivity in digital pictures, Journal of the Association for Computer Machinery 17 (1) (1970) 146-160.

9. A. Rosenfeld, Arcs and curves in digital pictures, Journal of the Association for Computer Machinery 20 (1) (1973) 81-87.

10. C. Ronse, A topological characterization of thinning, Theoretical Computer Science 43 (1) (1986) 31-41.

11. A. Rosenfeld, A characterization of parallel thinning algorithms, Information and Control 29 (3) (1975) 286-291.

12. T. Y. Kong, R. Litherland, A. Rosenfeld, Problems in the topology of binary digital images, in: J. van Mill, G. Reed (Eds.), Open Problems in Topology, Elsevier Science Publishers B.V. (NorthHolland), 1990, Ch. 23, pp. 377-385.

13. V. A. Kovalesky, Finite topology as applied to image analysis, Computer Vision, Graphics, and Image Processing 46 (2) (1989) $141-161$.

14. T. Y. Kong, Topology-preserving deletion of 1's from 2-, 3- and 4-dimensional binary images, in: E. Ahronovitz, C. Fiorio (Eds.), Discrete Geometry for Computer Imagery - DGCI'97, 7th International Workshop, Proceedings, Vol. 1347 of Lecture Notes in Computer Science, Springer, Montpellier, France, 1997, pp. 3-18.

15. G. Bertrand, On critical kernels, Comptes Rendus de l'Académie des Sciences, Série Mathématiques 1 (345) (2007) 363-367.

16. G. Bertrand, M. Couprie, Two-dimensional parallel thinning algorithms based on critical kernels, Journal of Mathematical Imaging and Vision 31 (1) (2008) 35-56.

17. N. Passat, L. Mazo, An introduction to simple sets, Pattern Recognition Letters 30 (15) (2009) 1366-1377.

18. P. Giblin, Graphs, surfaces and homology, Chapman and Hall, 1981. 
19. G. Bertrand, On topological watersheds, Journal of Mathematical Imaging and Vision 22 (2-3) (2005) 217-230.

20. E. C. Zeeman, Seminar on Combinatorial Topology, IHES, 1963.

21. T. Y. Kong, Minimal non-deletable sets and minimal noncodeletable sets in binary images, Theoretical Computer Science 406 (1-2) (2008) 97-118.

22. T. Y. Kong, On topology preservation in 2-D and 3-D thinning, International Journal of Pattern Recognition and Artificial Intelligence 9 (5) (1995) 813-844.

23. R. H. Bing, Some aspects of the topology of 3-manifolds related to the Poincaré conjecture, Lectures on Modern Mathematics II (1964) 93-128.

24. C. R. F. Maunder, Algebraic topology, Dover, 1996.

25. L. Mazo, N. Passat, On 2-dimensional simple sets in $n$-dimensional cubic grids, Discrete \& Computational Geometry (In press, doi: 10.1007/s00454-009-9195-x).

26. R. Malgouyres, A. Lenoir, Topology preservation within digital surfaces, Graphical Models 62 (2000) 71-84.

27. R. Klette, A. Rosenfeld, Digital Geometry, Morgan Kaufmann, 2004.

\section{Appendix}

Proof of Prop. 10 The forward implication is immediate, let us prove the converse. Suppose that $X$ is an extension of $Y$ and $\chi(Y)=\chi(X)$. Let $D=X \backslash Y$, and $k=|D|$. If $k=0$ then $Y=X$ and we are done. Suppose now that $k>0$, and suppose that the proposition holds for any complex $X^{\prime}$ instead of $X$, whenever $k^{\prime}<k$ (with $k^{\prime}=\left|X^{\prime} \backslash Y\right|$ ). We write $d_{Z}(x)$ to denote the number of 1 -faces of a complex $Z$ that include a given 0 -face $x$ of $Z$. Since each 1 -face includes exactly two 0 -faces, we have

$2\left|F_{1}(X)\right|=\sum_{x \in F_{0}(X)} d_{X}(x)=\sum_{x \in F_{0}(Y)} d_{X}(x)+\sum_{x \in F_{0}(D)} d_{X}(x)$.

Since $Y \leq X$, we have $d_{X}(x) \geq d_{Y}(x)$ for any $x$ in $F_{0}(Y)$. Since $k>0$, we know that $D \neq \emptyset$. Furthermore, we cannot have $\operatorname{dim}(D)=0$ because otherwise, $X$ would not be an extension of $Y$. Thus, there is at least one 1 -face in $D$. Let $X_{1}$ be a connected component of $X$ that contains at least one 1face of $D$. Since $X$ is an extension of $Y$, there is a connected component $Y_{1}$ of $Y$ that is included in $X_{1}$. Let $f \in X_{1} \backslash Y_{1}$ and $g \in Y_{1}$. Since $Y$ (hence also $Y_{1}$ ) is a complex, the first element $x$ of $Y_{1}$ in a path from $f$ to $g$ in $X_{1}$ is necessarily a 0face of $Y$, and $x$ is included in a 1 -face of $D$ by construction. We can see that $d_{X}(x)>d_{Y}(x)$. Thus, we have

$\sum_{x \in F_{0}(Y)} d_{X}(x)>\sum_{x \in F_{0}(Y)} d_{Y}(x)=2\left|F_{1}(Y)\right|$.

From (1) and (2), we deduce

$2\left|F_{1}(X)\right|-2\left|F_{1}(Y)\right|>\sum_{x \in F_{0}(D)} d_{X}(x)$.

Since $X$ is an extension of $Y$, there is no 0 -face $x$ in $D=X \backslash Y$ such that $d_{X}(x)=0$.

Suppose that all free 0 -faces of $X$ (if any) are in $Y$. Then, any 0 -face $x$ of $D$ is such that $d_{X}(x) \geq 2$, hence

$\sum_{x \in F_{0}(D)} d_{X}(x) \geq \sum_{x \in F_{0}(D)} 2=2\left|F_{0}(D)\right|=2\left|F_{0}(X)\right|-2\left|F_{0}(Y)\right|$.
From (3) and (4), we derive $\left|F_{1}(X)\right|-\left|F_{1}(Y)\right|>\left|F_{0}(X)\right|-$ $\left|F_{0}(Y)\right|$, i.e., $\left|F_{0}(X)\right|-\left|F_{1}(X)\right|<\left|F_{0}(Y)\right|-\left|F_{1}(Y)\right|$. Since $\operatorname{dim}(X \backslash Y) \leq 1$, for all $i \geq 2$ we have $\left|F_{i}(X)\right|=\left|F_{i}(Y)\right|$, hence $\chi(X)<\chi(Y)$, a contradiction.

From this, we deduce that $X$ has at least one free 0 -face $g$ that is not in $Y$, hence there is a free pair $(f, g)$ for $X$ such that $g \notin Y$ and $f \notin Y$ (otherwise $g$ would be in $Y$ since $Y$ is a complex). Let $X^{\prime}=X \backslash\{f, g\}$. By the recurrence hypothesis, $X^{\prime} \searrow Y$ and thus $X \searrow Y$. 